NASA Contractor Report 198439

$1 N-74$
33187

\title{
Fiber-Optic Based Compact Gas Leak Detection System
}

Wim A. de Groot

NYMA Inc.

Brook Park, Ohio

December 1995

Prepared for

Lewis Research Center

Under Contract NAS3-27186

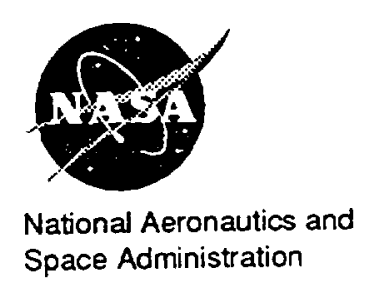





\title{
Fiber-Optic Based Compact Gas Leak Detection System
}

\author{
Wim A. de Groot \\ NYMA Inc. \\ Engineering Services Division \\ Brookpark, Ohio
}

\begin{abstract}
A propellant leak detection system based on Raman scattering principles is introduced. The proposed system is flexible and versatile as the result of the use of optical fibers. It is shown that multiple species can be monitored simultaneously. In this paper oxygen, nitrogen, carbon monoxide, and hydrogen are detected and monitored. The current detection sensitivity for both hydrogen and carbon monoxide is $1 \%$ partial pressure at ambient conditions. The sensitivity for oxygen and nitrogen is $0.5 \%$ partial pressure. The response time to changes in species concentration is three minutes. This system can be used to monitor multiple species at several locations.
\end{abstract}

\section{Introduction}

Propellant leakage is a concern for all aerospace systems using cryogenic, liquid, or gaseous propellants. of these gaseous propellant leaks, those involving hydrogen typically present the most urgent problem. Hydrogens' volatility makes it more difficult to contain. Furthermore, hydrogen leaks in air form a highly explosive mixture and therefore require immediate attention. Leakage of other fluid systems, although not as severe, could also pose significant carcinogenic or toxic hazards and should be addressed at the same level of urgency.

Implementation of an inexpensive, accurate, and fast leak detection system can increase mission safety, improve reliability, and reduce operating cost. The Space Shuttle, for example, utilizes liquid hydrogen in its main propulsion system. As of December 1993, 165 cumulative days of launch delays were due to hydrogen leaks or hydrogen leak sensor malfunction. Leaks from the 20 gaseous and liquid Space Shuttle fluid systems and

\footnotetext{
- Sr. Research Engineer, Member AIAA
}

Copyright (e) 1995 by NYMA, Inc. Published by the American Institute of Aeronautics and Astronautics, Inc. All rights reserved. associated servicing and ground support fluid systems combined, including the hydrogen propellant system, are currently detected using 11 different detection methods. These different leak detection systems introduce considerable complexity and are extremely labor intensive. Replacement of a number of these methods by a single alternative would improve safety, reliability, and economy.

In the case of the Space Shuttle and other hydrogen based systems, hydrogen detection is of paramount importance and most researchers have focused on this problem. Hunter ${ }^{1}$ recently analyzed commercially available hydrogen sensors and concluded that they do not meet requirements for aerospace applications. In a followup analysis ${ }^{2}$ of experimental hydrogen sensors two promising techniques were identified for further development. These are a metal-oxide-semiconductor (MOS) structure and a catalytic resistor sensor. Both are point contact sensors and based on the interaction of hydrogen with Palladium (Pd) or a Palladium alloy. The development of Pd sensors, their application, and current development status are described in two subsequent papers. ${ }^{3,4}$ The advantages of these two sensor types are 1) wide hydrogen concentration range coverage (from ppm to $100 \%$ ), 2) potential for microfabrication, and 3) low power requirements. Disadvantages include 1) the sensitivity and recovery time depend on the environment, 2) damage to the sensors can occur at high hydrogen concentrations and at higher temperatures, and 3) these sensors are limited to a single species.

This paper describes an alternative, noncontact leak detection method based on Raman scattering. Several researchers have pursued the use of this technology for hydrogen leak detection. ${ }^{5,6}$ Disadvantages of previous systems are that they are not in-situ, not real time, or 
bulky. The use of fiber optics, as proposed in this paper, mitigates these issues.

The Raman scattering principle is based on an energy exchange between light and internal molecular energy levels. Some laser light incident on molecules is scattered at frequencies which depend on the type of molecule. Scattered light intensity depends on the incident laser intensity and the number of molecules only. It is independent of temperature or the presence of other species.

Raman scattering by gases is a process which generates extremely weak emission. By comparison, Rayleigh scattered light is three orders of magnitude stronger, and fluorescence is about six to nine orders of magnitude stronger. These processes, however, do not provide the species specific information required in leak detection applications. In a Raman system, hardware and data acquisition software are designed to detect such a weak scattering and to separate it from other, much stronger radiation. Important considerations in the design of all leak detection systems are sensitivity, response time, system size and weight, power requirements, and cost.

Another design issue is the versatility of the detection system. Use of optical fibers for laser beam delivery and signal collection allows the use of many small probe heads. Multiple species can be detected simultaneously at each location. Results are presented in this paper for oxygen, hydrogen, nitrogen, and carbon monoxide. Multiple locations can be monitored by means of multiplexing the fibers.

\section{Theory}

Much of the theoretical background required for the development of a Raman based system was given in a previous paper. Incident laser light is focused, in-situ, into the gas to be analyzed. Light scattered from the gas molecules is collected and analyzed, with the collection optics determining spatial resolution.

The bulk of the scattered radiation has the same frequency as the incident laser frequency. This is Rayleigh scattering. A small portion, however, is scattered at different frequencies. This is the result of the coupling of the incident electromagnetic field and the rotationalvibrational modes of the molecules. This is
Raman scattering. The resulting radiation contains frequency components characteristic of the rotational vibrational structure of those molecules. These frequencies are shifted from the laser frequency by an amount directly proportional to the energy difference between two adjacent vibrational energy levels, and this is termed the Raman shift. Each frequency is species specific and so can be used to identify and quantify different gas constituents.

Because the intensity of the scattered light at each Raman shifted frequency is directly proportional to number density of the scattering species, the number density of that species can be determined from the Raman signal. Species partial pressures, therefore, can be measured assuming temperature and pressure are known. Alternatively, if temperature and pressure are unknown but constant, fluctuations in the gas composition can be monitored in real-time.

The Raman scattered line intensity, also referred to as line strength, depends not only on the number of molecules, but also on the polarization properties of the incident and scattered light, the geometry between incident and observed beams, and the rotationalvibrational transition probabilities. For a given geometry and polarization, the line intensities can be calculated from wavefunctions and the polarizability characteristics of the individual molecular species. 9 This is a straightforward task for diatomic molecules of known molecular properties. For many polyatomic molecules, these data are not known. The best alternative is to use empirical data, given in the form of Raman scattering cross sections, and to correct for the effect of polarization, laser wavelength, and geometry. ${ }^{10,11}$

Calculations are not necessary if a suitable calibration standard is available, in which the line strength is measured at a few different partial pressures of each molecular species. Because the line strength is linearly proportional to number density, the polarization and geometry parameters cancel. The transition probabilities for different molecular species follows from the line strengths at the different Raman shifted frequencies. During an experiment, different spectral lines are monitored and related to the calibration standard, from which the individual species number densities can be extracted. This is the approach that is taken in this research. 


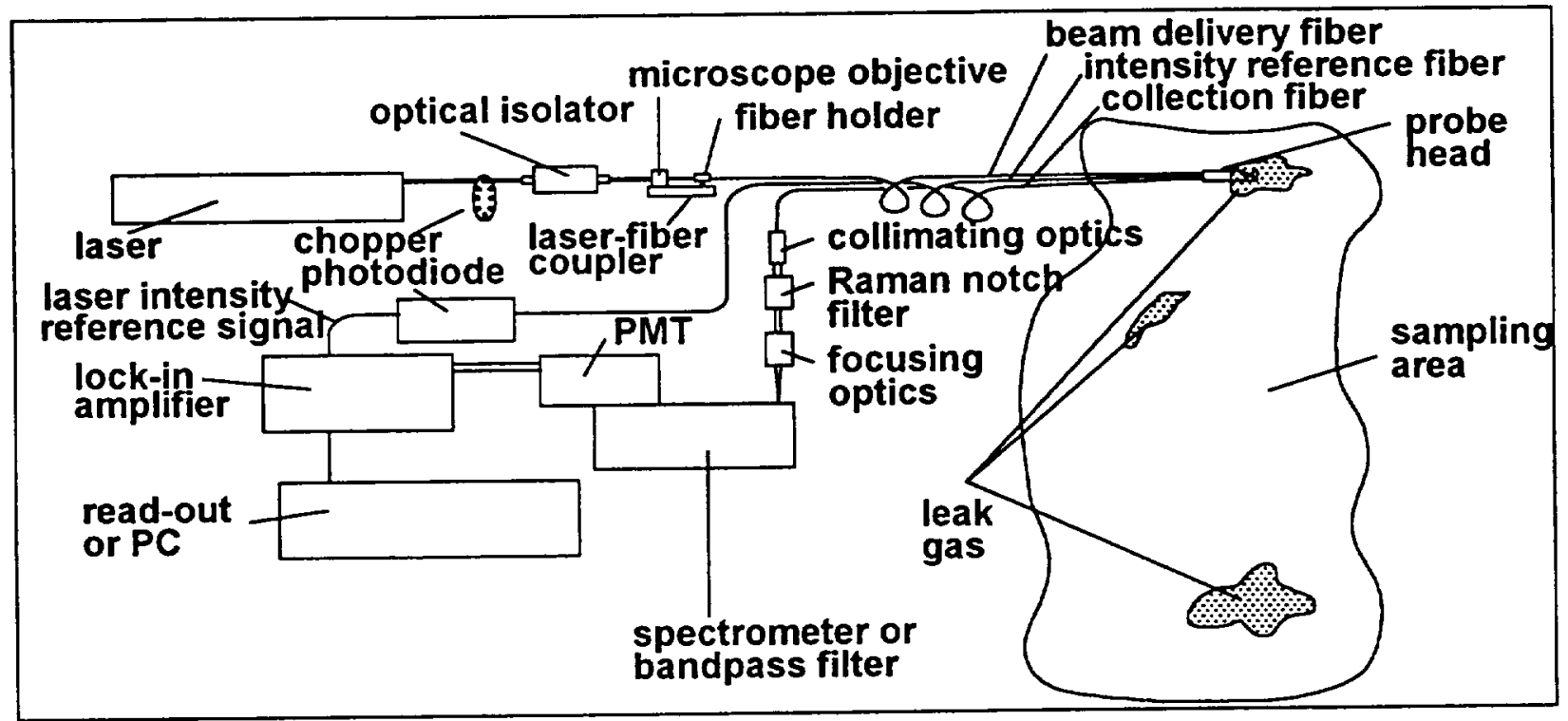

Figure 1: Raman leak detection data acquisition schematic

\section{Experimental Facility}

The experimental configuration used is shown in Figure 1 . The light source is a $5 \mathrm{~W}$ argon-lon laser which generates a vertically polarized, multimode, green beam with a wavelength of $514.5 \mathrm{~nm}$. The beam power is $700 \mathrm{~mW}$. This beam is modulated by means of a mechanical chopper with a frequency range from 0 to $500 \mathrm{~Hz}$. The beam then passes through an optical isolator (a Faraday rotator) to prevent destabilizing reflections back into the laser cavity.

A laser-fiber coupler utilizes a microscope objective to couple the beam into a $8.8 \mu \mathrm{m}$, single mode optical fiber. This $7 \mathrm{~m}$ long fiber guides the beam to the optical probe, which is shown in Figure 2. The fiber used in this research does not maintain polarization and so the exiting beam will be randomly polarized. A $1.8 \mathrm{~mm}$ diameter graded index lens collimates the light emerging from the fiber. The resulting beam diameter is $0.7 \mathrm{~mm}$ with a divergence of less than $3 \mathrm{mrad}$. A short pass filter with a cutoff frequency at $530 \mathrm{~nm}$ is placed downstream of the graded index lens. This filter transmits the laser beam and eliminates higher frequency light generated inside the delivery fiber by such processes as fluorescence and stimulated Raman scattering.
Two dichroic mirrors placed at 45 degrees with respect to the optical axes, and perpendicular to each other add to the rejection of higher frequency signals. They are designed to transmit the laser light while rejecting light with frequencies from 540 to $670 \mathrm{~nm}$. They are optimized for a 45 degree angle. The laser beam is displaced from the optical axis by the first dichroic mirror, and returned by the second, identical mirror.

A f/1.6 biconvex lens focuses the beam into the sample area. The same lens collects and collimates a portion of the light scattered from the molecules inside the sample. The second dichroic mirror separates this light, which propagates in the opposite direction of the incident laser beam, by wavelength. Laser light scattered from solid surfaces and the focusing lens, as well as Rayleigh scattered light from the sample molecules, which has the same frequency, will pass through the dichroic mirror. Light at higher frequencies, including the Raman scattered light is reflected at a 90 degree angle into a receiving assembly. A mask is placed between the incident and receiving assemblies which blocks off the center $2 \mathrm{~mm}$ to minimize laser light reflections from the dichroics and focusing lens. 


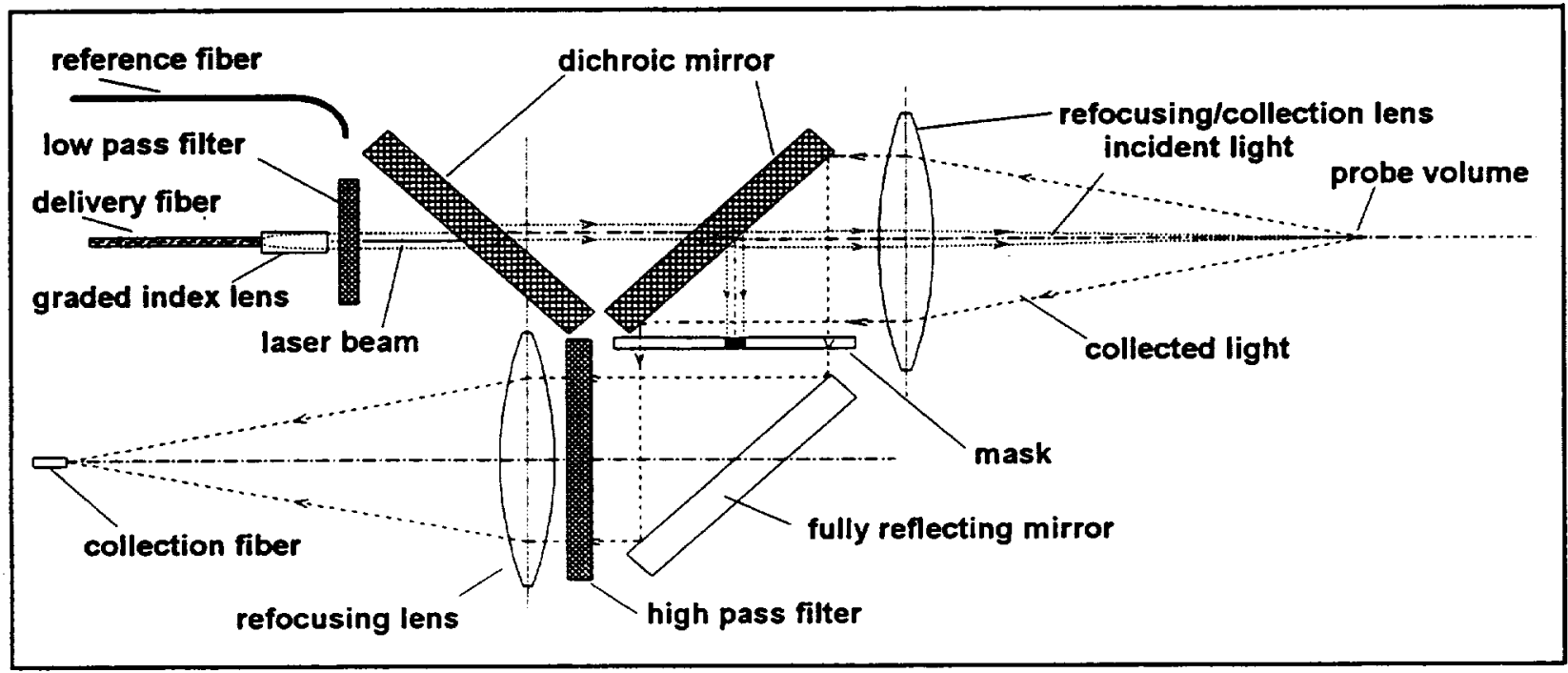

Figure 2: Raman gas detection diagnostics probe head

The receiving assembly consists of a long pass filter with a cut-off frequency at $530 \mathrm{~nm}$. Remaining laser light frequency components might still contaminate the collected Raman signal by generating fluorescence and stimulated Raman scattering inside the receiving fiber. The long pass filter prevents this. A front face mirror reflects the remaining collected light by 90 degrees parallel but opposite to the direction of the incident beam. It is focused by a $25 \mathrm{~mm}$ diameter, $80 \mathrm{~mm}$ focal length lens into a $7 \mathrm{~m}$ long, $200 \mu \mathrm{m}$ diameter optical fiber. Of the tested fibers with diameters of $8.8,100,200$ and $400 \mu \mathrm{m}$ this fiber diameter provided the best scattered light collection and transmission. This fiber guides the collected light to the data aquisition equipment.

The optical probe is $30 \mathrm{~mm}$ high, $60 \mathrm{~mm}$ wide, and $120 \mathrm{~mm}$ long. Figure 3 shows the optical probe in operation. The narrow green beam is the laser probe, while the red cone left of the waist represents that part of the Raman scattered light collected by the receiving optics.

As shown in Figure 1, light from the receiving fiber is collimated and passed through an optical notch filter and a holographic notch filter. Both filters are centered around $514.5 \mathrm{~nm}$ and designed to remove extraneous laser light.
Tests show that both filters are necessary to obtain optimum system performance.

A plano-convex lens with a f-number of 4 focuses the light into a 0.5 meter spectrometer with a 300 groove per $\mathrm{mm}$ grating and the same $f$-number. The spectrometer can scan a range of frequencies or be positioned at a single frequency. Spectrometer inlet and exit slit are set to $300 \mu \mathrm{m}$. This width is the optimized value that provides the maximum signal coupling from the $200 \mu \mathrm{m}$ collection fiber to the spectrometer, while maximizing the signal to noise ratio (SNR).

The Raman Shifts (R.S.) of the gases of interest in this investigation are $1556 \mathrm{~cm}^{-1}$ for oxygen, $2143 \mathrm{~cm}^{-1}$ for carbon monoxide, 2331 $\mathrm{cm}^{-1}$ for nitrogen, and $4156 \mathrm{~cm}^{-1}$ for hydrogen. With a laser wavelength at $514.5 \mathrm{~nm}$, the spectral locations of Raman scattered lines for these gases are 559.3, 578.3, 584.6, and 654.6 $\mathrm{nm}$, respectively. The spectral range of interest, therefore, is from 550 to $662 \mathrm{~nm}$.

An additional fiber is used to observe fluctuations in the laser power at the probe. One end of the fiber is mounted near the graded index lens, where it collects stray laser light. The other end is attached to a photodiode. The 
photodiode signal is processed with an electronic circuit containing a variable low pass filter, which removes the square wave modulation of the chopper. Thus, a proportional voltage used to eliminate the effect of laser intensity fluctuations on the Raman scattering signal is produced.

A multi-alkali photomultiplier (PMT) tube with a quantum efficiency of $12 \% @ 600 \mathrm{~nm}$, is mounted to the exit plane of the spectrometer. The PMT detects the light and provides a current proportional to the intensity. A cooled housing is used for the PM tube in order to reduce the dark noise on the signal. The output current from the PM tube is converted to a voltage via a $1 \mathrm{k} \Omega$ resistance. The resulting voltage is processed by a lock-in amplifier. A reference signal from the mechanical chopper is used as an external reference required to extract the signal from the noise.

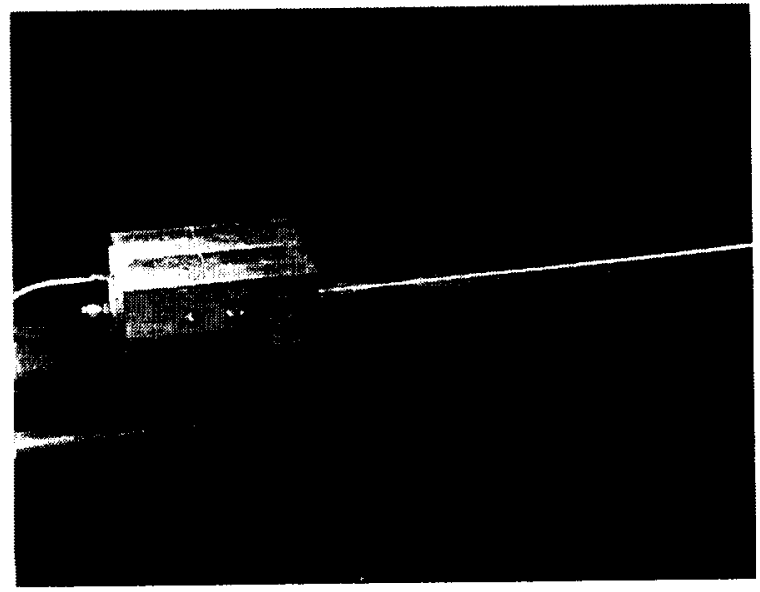

Figure 3: Optical probe in operation

To characterize and calibrate the detection system, the optical probe was mounted to a calibration vessel, shown in Figure 4. This calibration vessel can be pumped to $10^{-7} \mathrm{~Pa}$. Two optical quality windows are placed at 180 degrees for optical monitoring. The laser beam passes through the vessel and is captured in a beam dump to reduce stray laser light. A pressure gage indicates the vessel pressure. The vessel can be filled with a gas at any partial pressure from $10^{-7}$ up to $1.3 \times 10^{5} \mathrm{~Pa}$, limited by the optical windows. Gases or gas mixtures are limited to non-carcinogenic and non-explosive.

\section{System Considerations}

The most important operating parameters of a leak detection system are its sensitivity, response time, signal dependence on temperature and ambient atmosphere, and long term stability. These parameters were in detail investigated for the current Raman scattering system. In addition, the potential for simultaneous multispecies detection was analyzed. Hydrogen, nitrogen, oxygen, and carbon monoxide gases were the test gases of interest.

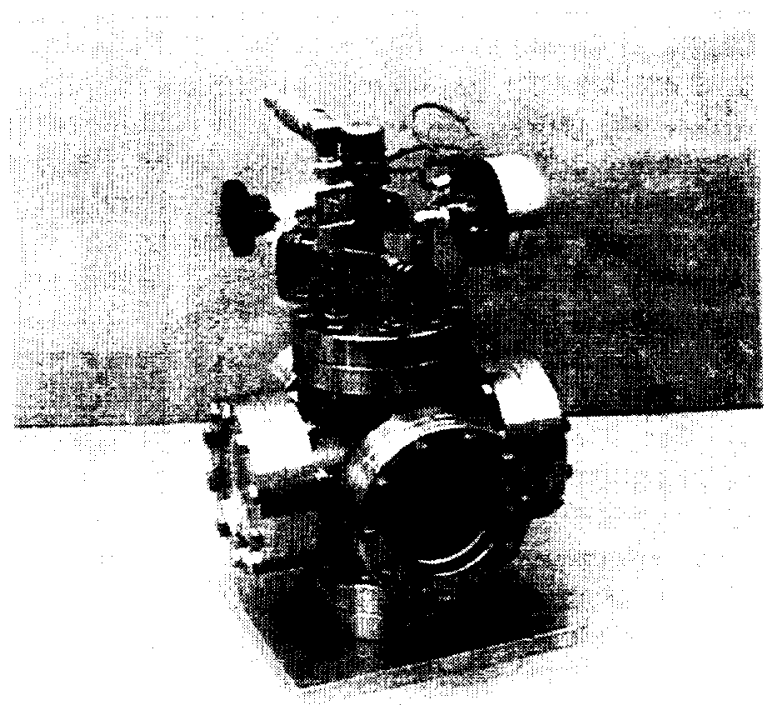

Figure 4: Test vessel with pressure gage

The measurement approach relies on the linear relationship between fluctuations in the Raman signal intensity at specific spectral locations and fluctuations in the number densities of the molecular species. The signal intensity provides a direct number density value assuming that the species independent background contribution does not vary. The effect of a constant background can be mitigated by calibration. A fluctuating background poses great difficulties. The source of the background and its effect are discussed in the next section.

\section{Background}

In addition to the usual broadband noise associated with optical systems, wavelength dependent contributions appear as a result of the optical design. Light transmission in optical fibers, especially high intensity laser light, generates secondary emission inside the fiber 
crystal structure. The most dominant signals are generated by means of fluorescence and stimulated Raman processes. Emissions of this type are usually broadband and incident intensity dependent, and can be several orders of magnitude stronger than the Raman scattering intensity from the gas molecules. The lock-in amplifier is not able to eliminate these signal contributions because they will have the same modulation frequency as the chopped laser beam

The detection system is designed to remove these emissions, mostly by means of optical filters. These optical filters, however, have their own wavelength dependent transmission curve, which will reveal itself in the test results as a frequency dependent background level. Factors influencing the intensity of this background level are length of optical fibers, polarization and intensity of the transmitted laser light, and number and type of filters.

Optical filters can be most effectively applied if the transmitted light has a specific polarization. Because the fibers scramble the polarization, however, use of optical filters is less effective. The dashed line in Figure 5 shows a background intensity curve as a function of wavelength for the full range of interest. The horizontal axis shows the wavelength, the vertical axis indicates the background intensity measured by the photomultiplier at each wavelength setting.

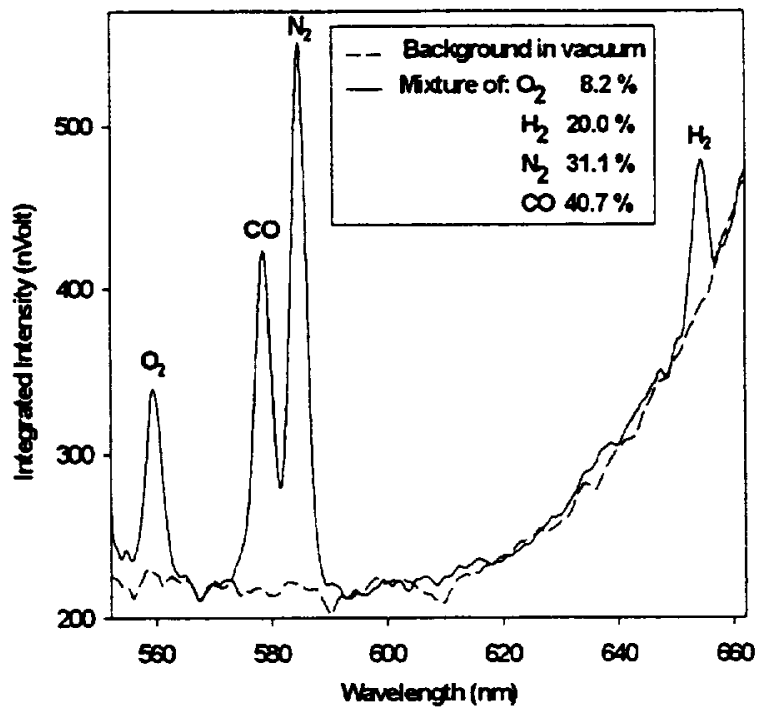

Figure 5: Wavelength scan: $a$. in vacuum; b. mixture of $\mathrm{H}_{2}, \mathrm{~N}_{2}, \mathrm{O}_{2}$, and $\mathrm{CO}$ inside vessel
The dashed line is the baseline noise of the detection system, including optical noise and electronic noise. This curve was generated by scanning from 550 to $662 \mathrm{~nm}$ with the probe monitoring conditions inside the test vessel at a pressure of $10^{-7} \mathrm{~Pa}$. The scan rate of the spectrometer for this curve is $0.05 \mathrm{~nm} / \mathrm{sec}$. The total scan took $38 \mathrm{~min}$. The time constant of the lock-in amplifier is $3 \mathrm{sec}$, which means that the full value of the signal is reached in $15 \mathrm{sec}$. Therefore, there is a certain delay between intensity changes and the detection of this change.

The solid curve in Figure 5 is generated under identical conditions as the background curve, with the exception that the test vessel is filled with a gas mixture, the composition of which is indicated in the figure legend. The full curve is a composite of two measurements. One measurement is taken in a mixture of carbon monoxide in air and the second measurement is taken with hydrogen only. The species partial pressures are selected such that the total pressure in the vessel is $10^{5} \mathrm{~Pa}(1 \mathrm{~atm}$.). Comparing the solid and dashed curves in this figure shows that the background is not affected by changes in gas conditions.

Both curves show a slope both at the low end $(550-560 \mathrm{~nm})$ and the high end $(610-660$ $\mathrm{nm}$ ) that are representative of the transmission curves of the dichroic mirrors. As yet, no solution has been found to fully eliminate this shape. The nitrogen and carbon monoxide lines are not affected. The oxygen line is slightly affected by having a sloping baseline, and the hydrogen line is strongly skewed due to this slope. Background subtraction techniques have not provided an acceptable solution due to the fact that the slope varies with changing laser intensity.

The locations of the individual Raman lines are slightly shifted with respect to the calculated values. The likely cause of this shift is experimental inaccuracy and the uncertainty introduced by the time delay of the lock-in amplifier. $A$ wavelength calibration with a mercury lamp verified these shifted spectral locations. The experimental maximum is easily located by scanning the pertinent wavelength range.

The peaks are relatively broad as the result of the wide spectrometer slits. Because the 
measurement strategy is based on observing the line intensity, the maximum signal strength is obtained by locating the spectrometer at the peak value of these spectral lines. Spectral resolution was not an issue, although the two lines of carbon monoxide and nitrogen did overlap. At each line center, the neighboring line does not contribute.

A change in laser power will affect the slope of the background curve. Increasing the laser power transmission through the fiber will lead to a non-linear amplification of the background with wavelength. This is shown in Figure 6 for intensity ratio of about 2 . For this, two scans of the hydrogen Raman lines were taken with incident intensities of 53 and $101 \mathrm{~mW}$. The resulting intensity curves were nondimensionalized with respect to the incident intensity. The curve for the higher laser power showed a lower magnitude than the lower laser power curve. This was caused by the nature of the background. During testing it was observed that the broad background contribution, which is due to electronic noise and ambient contributions, (approx. $200 \mathrm{nV}$ in Figure 5) does not change appreciably with changing incident power, whereas the wavelength dependent curve does change. It is not possible to separate these two contributions. When a straightforward incident intensity correction is applied to the full background, including the non-incident intensity dependent part, the curves are biased towards the lower intensity.

During leak detection tests, a direct compensation for the laser intensity fluctuations is applied to the monitored peak value. These compensations will create the same bias towards the lower laser intensity, indication a higher concentration then actually exists. Currently, a correct method for laser intensity correction has not been found.

It is noted that this wavelength dependent curve strongly resembles the transmission curves of the dichroic filter, indication that the origin of that part of the background is the delivery fiber. The use of short pass filters at the exit of this fiber does not attenuate this background. A strongly varying incident intensity therefore, even corrected for intensity fluctuations, will cause a large measurement inaccuracy.
In order to minimize the effect that this nonlinear amplification has on the measurements, care is taken to keep the laser power within a close range. Long term laser pointing instability, resulting in a varying laser-fiber coupling, does cause a drift in the transmitted power and accordingly in the slope of the background.

Current efforts are directed towards background subtraction. For the full scan from 643 to 662 , background subtraction can be done during post-processing of the data. For the currently proposed measurement approach, where only the Raman line intensity at the peak is observed, a real-time subtraction is needed. To that end, the background intensity at 654.2 $\mathrm{nm}$ is recorded as a function of intensity. Effects of the presence of windows or walls has to be accounted for. No satisfactory real-time subtraction method has been found yet.

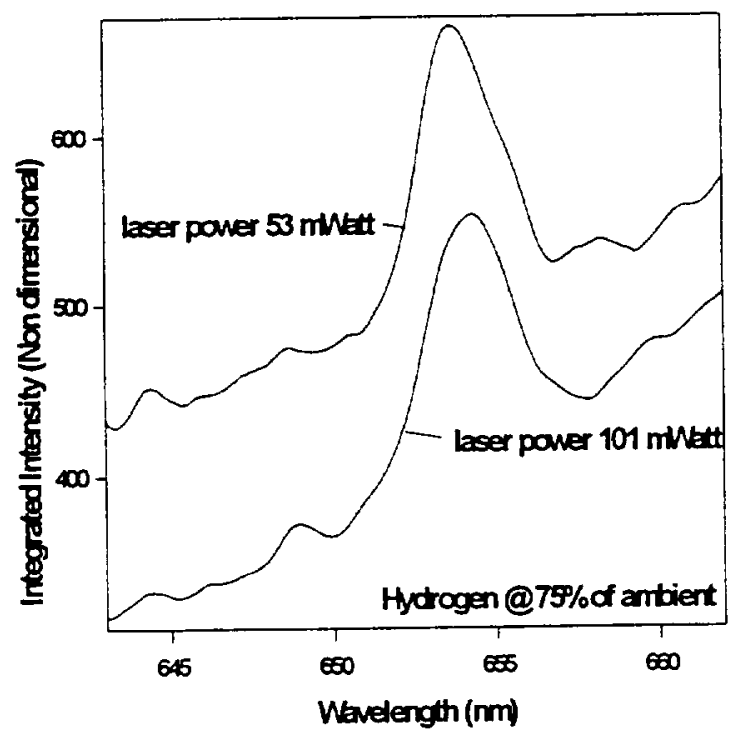

Figure 6: Effect of background as function of incident power

Tests were performed to investigate the effect of different fiber lengths on the background intensity curve. The $7 \mathrm{~m}$ long delivery fiber was replaced by a $3.5 \mathrm{~m}$ long fiber. The background scan was repeated and compared to the reference curve. A similar change was detected as shown in Figure 6 . The $7 \mathrm{~m}$ long fiber generated a stronger background signal, with a steeper slope. No straightforward correction to account for fiber length was found. Further investigation into the relationships between background and the various system parameters is needed. Methods to reduce the 
background and improve the signal to noise ratio are currently pursued. Replacement of the dichroic filters by holographic filters are expected to yield the greatest benefit.

Therefore it was concluded that the system configuration is important. Change in configuration can easily be handled, however, via the use of system transfer parameters.

\section{Results}

Sensitivity

Sensitivity measurement was done by means of testing with a fixed gas mixture inside the test vessel. Hydrogen measurements were taken at partial pressures of 0,1 and $2.8 \%$ of ambient, and from $10 \%$ up to $100 \%$ of ambient in steps of $10 \%$. The spectral range from 640.0 to $662.0 \mathrm{~nm}$, which includes the hydrogen Raman line, was scanned at least seven times for each concentration.

Data from these scans are displayed in Figure 7 , in which the wavelength is plotted on the horizontal axis and the intensity proportional voltage on the vertical axis. Each curve represents the average of 7 spectral scans. The sloping base line is caused by the background level. The slight differences in slope are the result of slightly varying laser intensities during the respective measurements. Efforts are currently ongoing to reduce the background contribution and to reduce the variance in the slope. It is expected that such an improvement will lead to a better SNR by reducing the background intensity.

Figure 7 shows that a concentration down to $1 \%$ of ambient can be detected. A change in $1 \%$ concentration will result in a change of $2.1 \mathrm{nVolt}$ in the signal. These curves are averaged, however. In order to verify the potential of detecting hydrogen concentration fluctuations among the fluctuations in the signal due to other sources, the intensity at the peak location, at $654.2 \mathrm{~nm}$ is monitored for $100 \mathrm{~min}$ for each concentration and the temporal fluctuations are recorded. A typical time trace is shown in Figure 8. The long observation time was chosen in order to eliminate long term signal drift. Changes in magnitude occur gradually, but peak to peak values give an uncertainty of $\pm 2 \%$. Current work is directed towards reducing this uncertainty.

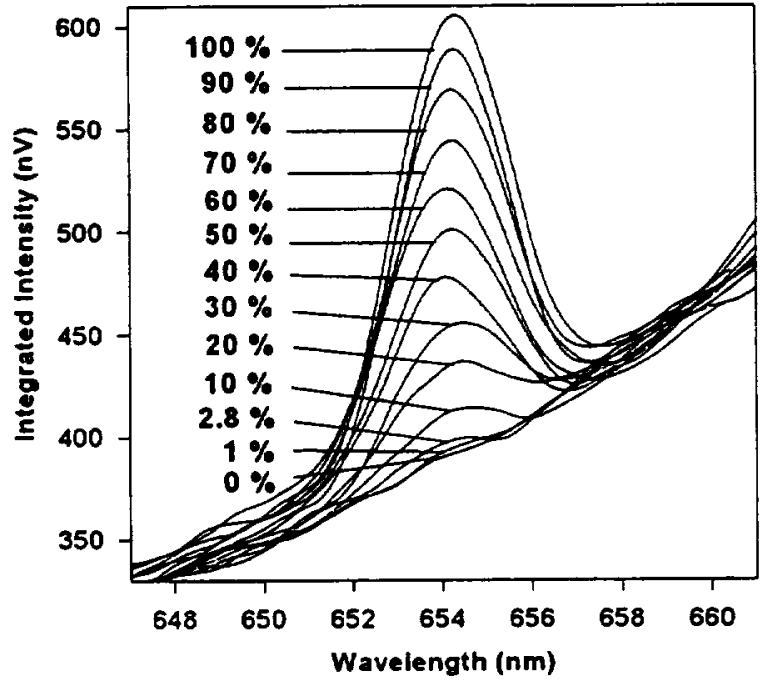

Figure 7: Hydrogen Raman line intensity as a function of concentration

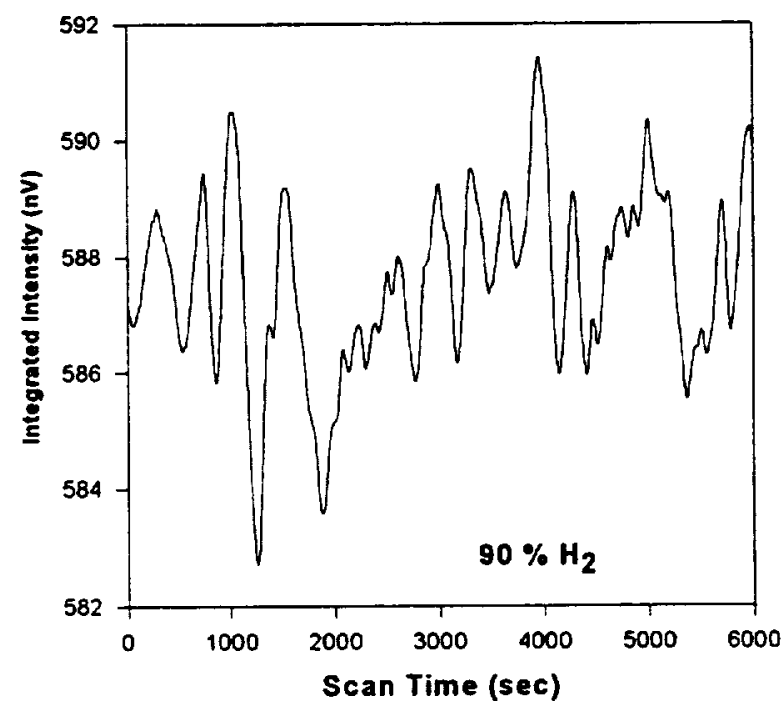

Figure 8: Typical time history of hydrogen concentration monitoring at constant concentration

Similar time histories are observed for all measured concentrations, and the mean values and standard deviation are determined. The results of these measurements are given in Figure 9. The horizontal axis represents the hydrogen partial pressure in \% of ambient pressure. This can be done directly because the temperature is constant. In that case, the pressure scales linearly with the measured number density, which is the quantity really being measured. The vertical axis indicates the 
signal voltage for each of these concentrations. The voltage at $0 \%$ hydrogen concentration represents the background at that spectral location. For each datapoint, the standard deviation of the fluctuations are given.

This figure shows that in the current configuration, a $1 \%$ concentration can be detected even though fluctuations in the signal make the uncertainty of the same magnitude. A more accurate determination requires less fluctuations. It is anticipated that fluctuations can be reduced by more accurately monitoring of the laser intensity fluctuations and by correcting for these fluctuations in real-time. The expected result with the current system would be a lower limit of hydrogen detectability of $1 \% \pm 0.5 \%$ of ambient.

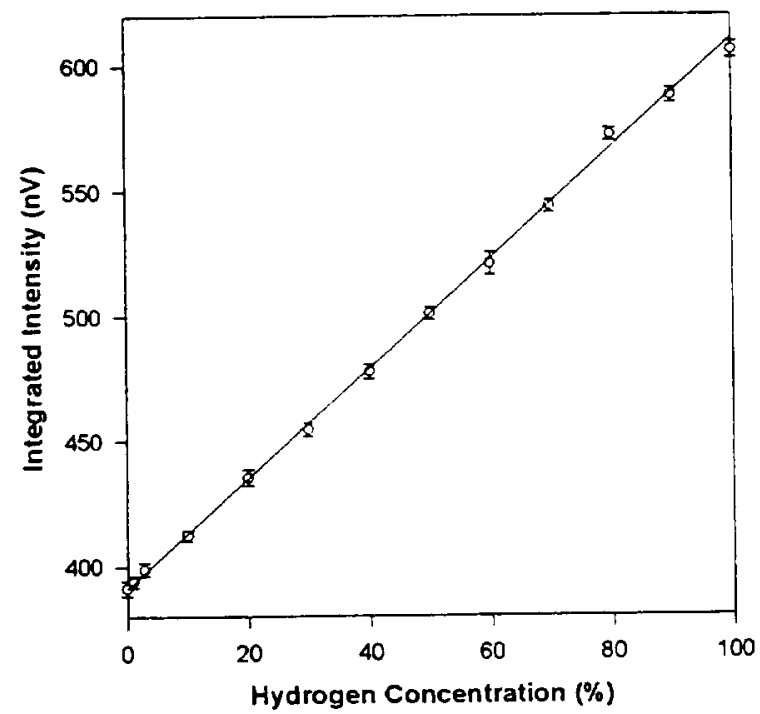

Figure 9: Calibration chart of system output versus hydrogen concentration

\section{Response Time}

The tests that are reported here were done with a lock-in amplifier constant of $30 \mathrm{sec}$. Because the amplifier is the slowest component in the system, it determines the system response time. Specifications for the amplifier give a time to reach the final value after a change in the signal of five times the time constant. This would bring the response time to the order of two to three min.

To verify that response time, the signal was monitored while the vessel was filled with a mixture of $2.1 \%$ hydrogen in air. The fill time was approximately $15 \mathrm{sec}$. The resulting signal is shown in Figure 10. Initially, the background signal in the vessel at vacuum was monitored for approximately one hour. This gave a background voltage of about 391 nVolt. After the gas mixture was inserted into the vessel, it took approximately $3 \mathrm{~min}$ to reach the final value. This is in agreement with the theoretical value. The uncertainty is caused by fluctuations, which make the definition of final value rather vague.

A clear change in mean value, agreeing with the change in hydrogen concentration was noticeable for this concentration, but the fluctuations do make a fast and accurate determination at these concentrations difficult. The focus of current research is to increase the signal level and reduce the fluctations.

\section{Stability}

An important consideration with the current system is the long term stability of the signal. Identified causes of instability are laser output, laser mode structure and laser-fiber coupling.

During the experiments reported in this paper it was found that long term laser pointing instability created a slow drift in laser intensity delivered to the probe head. The change in laser pointing over time caused the focal point of the microscope to drift from the center of the $8.8 \mu \mathrm{m}$ fiber. The result was a possible $50 \%$ decrease in laser intensity at the probe end over a time interval of 6 hours. A decrease was always observed, but the magnitude varied between experiments.

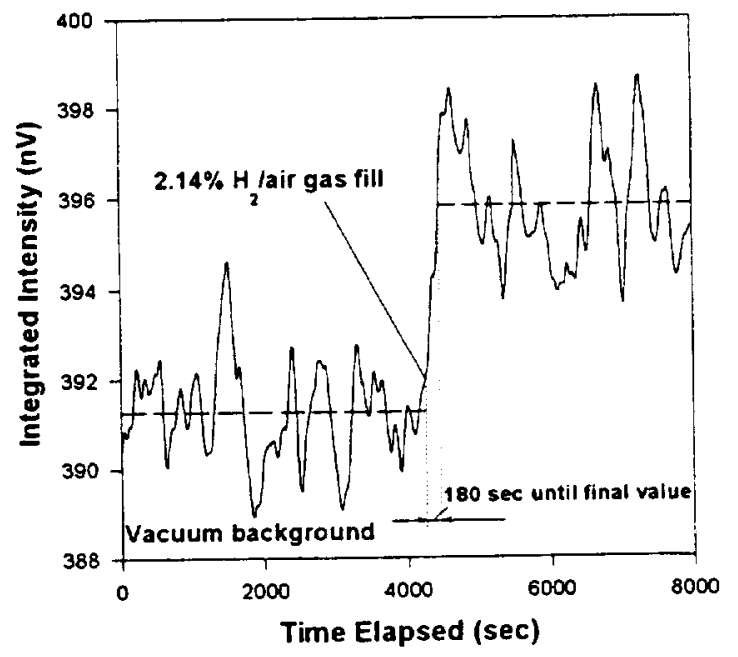

Figure 10: System response to concentration change 
Laser specifications indicate a $0.2 \%$ intensity fluctuation of the laser beam. Because of the age of the laser however, a much larger fluctuation, up to $3 \%$, was observed with a laser power meter. These intensity fluctuations are of the order of $1 \mathrm{~Hz}$. And because a time constant of $30 \mathrm{sec}$ is used, these laser fluctuations are not expected to contribute significantly to the signal fluctuations.

Another laser instability had a greater contribution to the signal fluctuations. Mode hopping causes the laser intensity distribution over the different laser modes to vary at random time intervals. The single mode fiber acts as a mode filter, transmitting only a limited number of modes. This mode hopping causes a random change in delivered laser intensity, with observed intensity jumps of up to $10 \%$. Use of a more stable laser will alleviate these problems.

Thus far, correction of the measured signal for laser power fluctuations has not been succesfull, but the development of a correction circuit is continuing.

\section{Ambient Atmosphere and Temperature Effect}

Based on theoretical considerations, change in ambient species besides the species to be measured will only affect the measured signal if the Raman shift of the species overlap. A monatomic species such as Helium, which could be present at launch sites for purging purposes, does not have vibrational or rotational energies, thus will not exhibit Raman scattering. Its presence will not affect the scattering signal. Oxygen and nitrogen, which could be present, have Raman shifts far from the hydrogen and, theoretically, will not adversely influence the measurement. To verify the above considerations, tests were completed with hydrogen at $2.8 \%$ partial pressure in vacuum, in $40 \%$ helium and in helium/air. The results showed that the presence of the tested "background" gases did not affect the hydrogen signal level.

A possible concern, which has not been investigated, is the presence of water droplets or alternate particulate matter. Light scattered by such particles (Mie scattering) is of the laser wavelength and consequently will mostly be filtered out by the optical notch filters. A strongly attenuated light signal does result. Therefore, these are not expected to contribute significantly to the signal, as long as the percentage of particulate matter is small ( < $1 \%$ ). A larger percentage of particles or droplets, however, could still contaminate the weak Raman signal. Furthermore, these particles displace the gas that is monitored. Therefore it is expected that large quantities of particulate matter will create data analysis problems.

\section{Multiple species monitoring}

One of the advantages of the Raman-based system is the capability of simultaneously monitoring several species. To achieve this capability, it is not necessary to scan the full wavelength range. Several options are available to monitor multiple gases.

A polychromator can disperse the Raman spectrum. Fiber optics placed at the exit plane of the polychromator to match the wavelengths of interest can transport the signals to photomultiplier tubes. This is a commonly used technique in the field of spectroscopy. PM tube signals can be monitored real time with lock-in amplifiers for species detection.

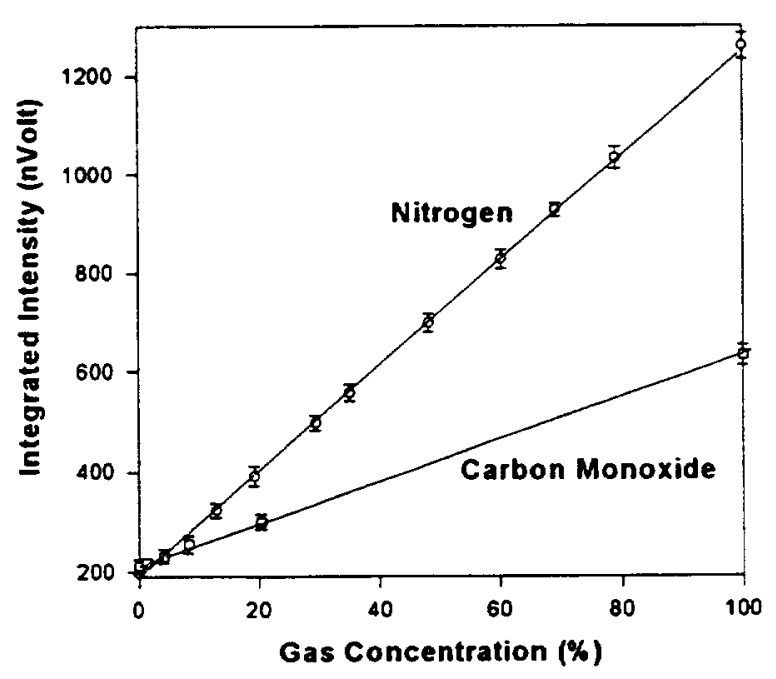

Figure 11: Nitrogen and Carbon Monoxide Calibration Chart.

A less expensive method involves the use of optical bandpass filters. The Raman signal could be multiplexed between different filter/PM tube combinations and monitored. This would be a slower method however and only quasisimultaneous. Switching between different filters can be accomplished in seconds. In the current configuration, however, the time constant of the 
lock-in amplifier limits the response to the new signal to several minutes.

The obtainable sensitivities for each gas has to be analyzed. The line strength of nitrogen and oxygen in the back scatter orientation is much stronger than that of hydrogen, which in turn is stronger than that of carbon dioxide. Line location also contributes to the line strength and PM tube sensitivity. Calibration curves for the current system have also been made for nitrogen and carbon monoxide. They are given in Figure 11.

This figure shows that the signal intensity for nitrogen is stronger than that for carbon monoxide. The obtainable sensitivity is therefore better for nitrogen. Both these gases have a better signal to noise ratio than hydrogen as the result of a much lower background noise.

Future tests have to show how many gases can be measured simultaneously and which of the methods described above will provide the optimum trade between simplicity, cost, and sensitivity.

\section{Size, Weight, and Power Consumption}

The current laser and data acquisition equipment cover an area of $4 \mathrm{~m}^{2}$. This equipment, however can be installed inside a room, with fibers extending to all locations to be monitored, making the system extremely flexible. The system could be improved by the selection of a $300 \mathrm{~mW}$ laser with the same power in the green beam, but less input power requirements.

The size of the optical probes is approximately $2.2 \times 10^{-3} \mathrm{~m}^{3}$ and can be made smaller. The weight is less than 200 grams. Optimizing the design can improve these numbers substantially. A smaller probe makes it easy to mount the probe in locations that have to be monitored or to carry the probe for field measurements in which the laser and data acquisition equipment are mounted in a briefcase.

The three optical fibers that carry the laser beam, and the Raman and reference signals do not require shielding. There is no if interference, which can interfere with electronic signals. Fibers are available that can withstand up to $700^{\circ} \mathrm{C}$. This system is therefore uniquely capable of monitoring hostile environments.
There is a limit, however, to the maximum fiber length. The best optical fibers have a transmission close to $99.9 \%$ transmission per meter for the used wavelength range. This causes a $40 \%$ drop in light intensity for fibers of $500 \mathrm{~m}$ length, both for the receiving and collection fibers. Whether this loss in intensity is acceptable depends on the required sensitivity.

Several configurations are possible. A centralized laser and data acquisition system with a powerful laser could reach out with fibers to monitor a number of locations simultaneously. The laser beam can be split into several beams of equal intensity, which are coupled to different fibers.

A more economical system would utilize a multiplexer. ${ }^{7}$ A multiplexer mechanically couples light from a single fiber into a number of fibers, each extending into a different location. A stepping motor positions the single fiber in front of any of available fibers in less then a second. Tests have shown that a coupling efficiency of $75 \%$ can be obtained with such a device. Measurements done with such a system are quasi-simultaneous and will have a longer response time. Dependent on the applications, this might or might not be acceptable.

The laser consumes the major part of the power needed. Dependent on the type of laser, the power requirements can easily be satisfied for ground based systems, but needs to be improved for in-flight systems.

\section{Cost}

The projected cost will also depend on the complexity and capability of the system. The cost of a large system, with centralized laser and data acquisition and processing facility, which will be able to detect multiple species at several locations can be expensive $(70-80 \mathrm{k} \$)$. A small, briefcase size system can be designed economically $(10-12 \mathrm{~K} \$)$. There will be a tradeoff however between cost, sensitivity, and response time.

\section{Conclusions}

A multispecies leak detection system based on Raman scattering is introduced. Advantages are the in-situ, real time measurement, the independence from environmental conditions, the small size, and the capability of detecting multiple species at several locations. 
Tests were performed on oxygen, nitrogen, carbon monoxide and hydrogen, both as single gases and as mixtures of gases. Helium was used as a filler gas to verify the independence of environmental conditions. Results from pure gases and mixtures of gases showed the independence of environmental conditions. The expected temperature range is from cryogenic to $700^{\circ} \mathrm{C}$. The effect of the presence of particles or droplets still has to be investigated.

Detection capabilities ranged from $1 \%$ to $100 \%$ for hydrogen and carbon monoxide, and from $0.5 \%$ to $100 \%$ for nitrogen and oxygen at ambient conditions. Other species still have to be tested. These sensitivities are comparable with the catalytic resistor sensors. Further development is expected to improve the sensitivity to $0.1 \%$.

The current response time to full final value is about $3 \mathrm{~min}$, which again is comparable to the catalytic resistor sensor. The time constant of the lock-in amplifier is the limiting factor. Faster response can be obtained at the expense of the sensitivity. An anticipated improvement in the signal fluctuations as the result of better compensation circuit will allow a faster time constant. As a result it is expected that the response time will be on the order of 5 to 10 sec.

\section{References}

"Hunter, G. W., "A Survey and Analysis of Commercially Available Hydrogen Sensors," NASA TM-105878, November, 1992.

${ }^{2}$ Hunter, G. W., "A Survey and Analysis of Experimental Hydrogen Sensors," NASA TM106300, October, 1992.

${ }^{3}$ Hunter, G. W., Neudeck, P. G., Liu, C. C., and $\mathrm{Wu}, \mathrm{Q}$. H., "Advances in Hydrogen Sensor Technology for Aerospace Applications," 1994 Conference on Advanced Earth-To-Orbit Propulsion Technology, NASA Marshall Space Flight Center, Huntsville, Alabama, May 17-19, 1994.

${ }^{4}$ Hunter, G. W., Bickford, R. L., Jansa, E. D., Makel, D. B., Liu, C. C., Wu, Q. H., and Powers, W. D., Microfabricated Hydrogen Sensor Technology for Aerospace and Commercial
Applications," NASA TM-106703, NASAVSPIE Symposium, San Diego, CA, July 24-29, 1994.

5Caputo, B., "Hydrogen Detection Lidar," Computer Genetics Corporation, VEN-1519, Final Report NASA Contract \#NAS10-11421, 1988.

${ }^{6}$ Adler-Golden, S. M., Goldstein, N., Bien, F., Matthew, M. W., Gersh, M. E., Cheng, W. K., and Adams, F. W., "Laser Raman sensor for measurement of trace-hydrogen gas," Applied Optics, Vol.31, No. 6, February 1992, pp 831835.

'de Groot, W. A., Myers, R. M., and Zube, D., "Fiber Optic Switch for Broadband Emission Spectroscopy", Laser Techbriefs, Vol. 2, No. 2, 1994, pp. 50-54.

${ }^{8}$ de Groot, W. A., "The Use of Spontaneous Raman Scattering for Hydrogen Leak Detection," AIAA 94-2983, Indianapolis, IN, June 27-29, 1994.

'James, T. C. and Klemperer, W., "Line Intensities in the Raman Effect of ' $\Sigma$ Diatomic Molecules," The Joumal of Chemical Physics, Vol. 31, No. 1, July 1959, pp. 130-134.

${ }^{10 S c h r o e t t e r, ~ H . ~ W ., ~ a n d ~ K l o e c k n e r, ~ H . ~ W ., ~}$ "Raman Scattering Cross Sections in Gases and Liquids," Raman spectroscopy of Gases and Liquids (A. Weber, Ed.) Topics in Current Physics, Springer Verlag, 1979.

\footnotetext{
${ }^{11 H i r s c h f e l d, ~ T ., ~ " C o r r e c t i o n ~ o f ~ R a m a n ~ C r o s s ~}$ Section from Laboratory to Remote Spectrometer Geometries," Applied Spectroscopy, Vol.27, No. 5, 1973.
} 


\begin{tabular}{|c|c|c|c|c|}
\hline \multicolumn{3}{|c|}{ REPORT DOCUMENTATION PAGE } & \multicolumn{2}{|r|}{$\begin{array}{l}\text { Form Approved } \\
\text { OMB No. 0704-0188 }\end{array}$} \\
\hline \multicolumn{5}{|c|}{ 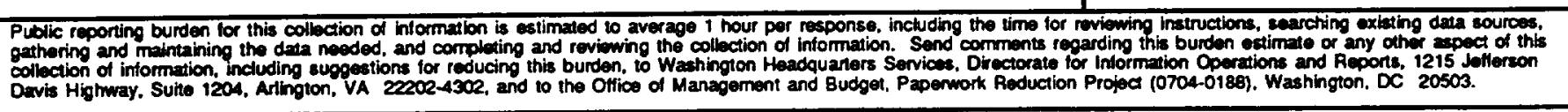 } \\
\hline 1. AGENCY USE ONLY (Leave blank) & $\begin{array}{l}\text { 2. REPORT DATE } \\
\text { December } 1995\end{array}$ & \multicolumn{3}{|c|}{$\begin{array}{l}\text { 3. REPOAT TYPE AND DATES COVERED } \\
\text { Final Contractor Report }\end{array}$} \\
\hline \multicolumn{3}{|c|}{$\begin{array}{l}\text { 4. TITE AND SUBTITLE } \\
\text { Fiber-Optic Based Compact Gas Leak Detection System }\end{array}$} & \multirow{2}{*}{\multicolumn{2}{|c|}{$\begin{array}{l}\text { 5. FUNDING NUMBERS } \\
\text { WU-242-70-02 } \\
\text { C-NAS3-27186 }\end{array}$}} \\
\hline \multicolumn{3}{|l|}{$\begin{array}{l}\text { 6. AUTHOR(S) } \\
\text { Wim A. de Groot }\end{array}$} & & \\
\hline \multicolumn{3}{|c|}{$\begin{array}{l}\text { 7. PERFORMANG ORGANIZATION NAME(S) AND ADDRESS(ES) } \\
\text { NYMA Inc. } \\
\text { 2001 Aerospace Parkway } \\
\text { Brook Park, Ohio } 44142\end{array}$} & \multicolumn{2}{|c|}{$\begin{array}{l}\text { 8. PERFORMMNG ORGANIZATION } \\
\text { REPORT NUMBER } \\
\text { E-10053 }\end{array}$} \\
\hline \multicolumn{3}{|c|}{$\begin{array}{l}\text { 9. SPONSORING/MONITORING AGENCY NAME(S) AND ADDRESS(ES) } \\
\text { National Aeronautics and Space Administration } \\
\text { Lewis Research Center } \\
\text { Cleveland, Ohio } 44135-3191\end{array}$} & 10. SP & $\begin{array}{l}\text { NSORINGMONTOAING } \\
\text { NCY REPORT NUMBER } \\
\text { SA CR-198439 } \\
\text { A-95-2646 }\end{array}$ \\
\hline \multicolumn{5}{|c|}{$\begin{array}{l}\text { 11. SUPPLEMENTARY NOTES } \\
\text { Prepared for the } 31 \text { st Joint Propulsion Conference and Exhibit cosponsored by AIAA, ASME, SAE, and ASME, San } \\
\text { Diego, Califomia, July } 10-12,1995 \text {. Project manager, Steven J. Schneider, Space Propulsion Technology Division, } \\
\text { NASA Lewis Research Center, organization code, (216) } 977-7484 \text {. }\end{array}$} \\
\hline \multicolumn{3}{|c|}{$\begin{array}{l}\text { 12a. DISTRIBUTIONAVAILABILTY STATEMENT } \\
\text { Unclassified - Unlimited } \\
\text { Subject Categories } 72 \text { and } 20 \\
\quad 74 \\
\text { This publication is available from the NASA Center for Aerospace Information, (301) 621-0390. }\end{array}$} & \multicolumn{2}{|c|}{ 12b. DISTRIBUTION CODE } \\
\hline \multicolumn{5}{|c|}{$\begin{array}{l}\text { A propellant leak detection system based on Raman scatter } \\
\text { and versatile as the result of the use of optical fibers. It is s } \\
\text { In this paper oxygen, nitrogen, carbon monoxide, and hydr } \\
\text { sensitivity for both hydrogen and carbon monoxide is } 1 \% \mathrm{p} \\
\text { oxygen and nitrogen is } 0.5 \% \text { partial pressure. The response } \\
\text { This system can be used to monitor multiple species at seve }\end{array}$} \\
\hline \multirow{3}{*}{\multicolumn{2}{|c|}{$\begin{array}{l}\text { 14. SUBJECT TERMS } \\
\text { Raman scattering; Leak detection }\end{array}$}} & & & $\begin{array}{c}\text { 15. NUMBER OF PAGES } \\
14\end{array}$ \\
\hline & & & & 16. PRICE CODE \\
\hline & & & & A03 \\
\hline $\begin{array}{l}\text { 17. SECURITY CLASSIFICATION } \\
\text { OF REPORT }\end{array}$ & $\begin{array}{l}\text { 18. SECURTY CLASSIFICATION } \\
\text { OF THIS PAGE }\end{array}$ & $\begin{array}{l}\text { 19. SECURITY CLASSIFICA } \\
\text { OF ABSTRACT }\end{array}$ & ATION & 20. LIMITATION OF ABSTRACT \\
\hline Unclassified & Unclassified & Unclassified & & \\
\hline
\end{tabular}


National Aeronautics and

Space Administration

Lewis Research Center

21000 Brookpark Rd.

Cleveland, $\mathrm{OH}$ 44135-3191

Official Business

Penalty for Privale Use $\$ 300$

POSTMASTER: If Undeliverable - Do Not Return 